Short Communication

\title{
In Silico Designing of a Multi-Epitope Based Vaccine Candidate Against Human Adenovirus Type B3 Respiratory Infections by Utilising Various Immunoinformatics Approaches
}

\author{
Somnath Panda ${ }^{1}$, Urmila Banik ${ }^{2}$ and Arun Kumar Adhikary ${ }^{1 *}$ \\ ${ }^{1}$ Unit of Microbiology, AIMST University, Faculty of Medicine, Jalan Bedong Semeling, 08100 Bedong, \\ Kedah, Malaysia \\ ${ }^{2}$ Unit of Pathology, AIMST University, Faculty of Medicine, Jalan Bedong Semeling, 08100 Bedong, Kedah, \\ Malaysia
}

\begin{abstract}
Human adenovirus type B3 (HAdV-B3) causes severe respiratory infections, hence an efficient vaccine is required. Unfortunately, the presence of numerous hexon variations makes conventional vaccine designing difficult which warrants an alternative method. Therefore, an in silico multi-epitope vaccine had been constructed against appropriate hexon variants of HAdV-B3. The allergenicity, antigenicity, structure, physicochemical properties along with molecular docking with TLR-3 and TLR-9 had also been predicted. The constructed vaccine had 23 different

ARTICLE INFO

Article history:

Received: 8 September 2020

Accepted: 27 November 2020

Published: 22 January 2021

epitopes. It showed non-allergic but antigenic nature with 30 hours of half-life in vitro and exhibited thermostable nature. We anticipate that this will considerably reduce the time and expense of biological work needed for future vaccine development.
\end{abstract}

DOI: https://doi.org/10.47836/pjst.29.1.32

E-mail addresses:

somnathpanda86@yahoo.co.uk (Somnath Panda)

urmila_banik@yahoo.co.in (Urmila Banik)

arun_adhikary@msn.com (Arun Kumar Adhikary)

*Corresponding author
Keywords: Human adenovirus type B3, hypervariable regions, immunoinformatics, multi-epitope vaccine construct 


\section{INTRODUCTION}

Human adenoviruses (HAdVs) possess a double stranded DNA without an envelope. As of today, 103 types of HAdVs had been categorized (http://hadvwg.gmu.edu/) in accordance with their serological and genetic criteria and accordingly are organized into seven groups (A-G). Human adenovirus type B3 (HAdV-B3) causes respiratory infections, epidemic keratoconjunctivitis, pharyngoconjunctival fever, acute gastroenteritis, and also occasionally involve the nervous system (Harley et al., 2001).

The pediatric age group, predominantly, neonates are considered to be the primary targets for nosocomial and community-acquired pneumonia caused by HAdV-B3. The pneumonia can lead to respiratory or heart failure with fatal outcome. As per example, the epidemic of adenoviral pneumonia that occurred in Northern China recorded a fatality of up to $30 \%$ and HAdV-B3 along with B7 were recognized as the major causative agents (Dong, 2005). Moreover, HAdV-B3 also causes severe illness among immunocompromised patients with increased mortality (Lai et al., 2013). During recent years, the increasing number of detection of HAdV-B3 from respiratory samples supports that it is the most predominant form of HAdVs accountable for respiratory tract infections (Chen \& Tian, 2018). It has been observed that HAdV-B3 could be responsible for up to $87 \%$ of all adenoviral respiratory infections (Lynch \& Kajon, 2016).

In spite of its medical and epidemiological significance, there is no targeted antivirals or vaccines against HAdV-B3. A number of studies on antivirals showed that nucleoside analogues and protease inhibitors have some activity against some types of adenoviruses. But their clinical usefulness is still not well-proven (Lion, 2014). Currently, the mainstay for the management of adenoviral infections is symptomatic. Thus, a vaccine that is economical, efficacious, and also safe against HAdV-B3 is required.

It is well-known that antibodies against HAdV-B3 hexon protein can counteract the infectivity of HAdVs in a type-specific manner (Pichla-Gollon et al., 2007). The capsid of HAdVs is mainly formed by hexon which includes three identical units of the polypeptide consisting of 945 amino acids (AA) and its hypervariable regions (HVRs) contain the epitopes (Liu et al., 2018) which are especially significant for designing vaccine and developing novel therapeutic approaches. Recently, we found out that the HVRs of HAdV-B3 hexon was highly variable, and based upon those variations, the available field strains had been categorized into 25 hexon variants, $3 \mathrm{Hv}-1$ to $3 \mathrm{Hv}-25$, (Haque et al., 2018). As a result of this vast heterogeneity, conventional vaccine development remains challenging.

To overcome this challenge, we hypothesized that a novel multi-epitope vaccine would be a more cost-effective method and with reference to our current findings, four hexon variants $(3 \mathrm{Hv}-1,2,3$, and 4$)$ were selected to form a multi-epitope vaccine construct (Panda et al., 2020). The objectives of the study were to predict suitable epitopes (B-cell 
epitopes, Helper T Lymphocyte, and Cytotoxic T Lymphocyte epitopes) from the HVRs of those major variants with the help of various immunoinformatics tools and to construct and analyze a multi-epitope vaccine that could potentially be investigated for additional biological work for future vaccine development.

\section{METHODOLOGY}

\section{HAdV-B3 Hexon Protein Sequence Selection for Vaccine Construct}

In the HAdV-B3 prototype, the hypervariable regions comprise 319 amino acids and extend from 132 to 450 amino acids on hexon. After multisequence alignment, the HVRs associated with $3 \mathrm{Hv}-1,2,3$, and 4 (the specific 319 amino acids long sequence), had been selected for further analysis. All the sequences (GenBank Accession Nos. AB330084, AF542106, DQ099432, KC456085, and AY854180), were gained from NCBI (National Centre for Biotechnology Information-http://www.ncbi.nlm.nih.gov/) and were analyzed further to predict the appropriate epitopes.

\section{Linear B Cell Epitope (BCE) Prediction}

$\mathrm{BCE}$ represents the antigen where $\mathrm{B}$ lymphocytes bind and carry out a fundamental role in vaccine construction. BCE were attained from BCEPRED (Prediction of B Cell Epitopes-http://crdd.osdd.net/raghava/bcepred/) database. It predicts according to the various physicochemical aspects of BCE and gives results with both graphical and tabular format. The graphical format assists the users in rapid visualization of BCE where the peaks above the default threshold (2.5) are considered as BCE. The tabular output gives a score established on various physical and biochemical aspects. The threshold (-3 to 3 ) including every other factor (hydrophilicity, accessibility, flexibility, antigenic propensity, and polarity) was fixed at default, and epitopes are indicated in blue color (Saha \& Raghava, 2006b). All obtained BCE were incorporated in the concluding vaccine construct.

\section{Helper T Lymphocyte (HTL) Epitopes Prediction}

HTL responses play a significant role in the initiation of both humoral and cellular immune responses. Therefore, HTL epitopes are likely to be a fundamental element of vaccines. For this reason, HTL epitopes of 15 AA long was attained from the IEDB (http://tools. iedb.org/mhcii/) database following IEDB recommended method 2.22 for the 7-allele HLA (Human Leukocyte Antigen) reference set. The IEDB (Immune Epitope Database) is a freely available resource funded by NIAID (National Institute of Allergy and Infectious Diseases-https://www.niaid.nih.gov). It catalogs experimental data on antibody and T cell epitopes studied in humans, non-human primates, and other animal species in the context of infectious disease, allergy, autoimmunity, and transplantation. It also hosts tools to 
assist in the prediction and analysis of epitopes. HTL selection was established on their $\mathrm{IC}_{50}$ values; the peptides having maximum affinity have $\mathrm{IC}_{50}<50 \mathrm{nM}$, peptides having intermediate affinity have $\mathrm{IC}_{50}<500 \mathrm{nM}$, and peptides holding $\mathrm{IC}_{50}<5000 \mathrm{nM}$ show the lowest amount of affinity, therefore the lowermost $\mathrm{IC}_{50}$ value demonstrates the highest affinity (Wang et al., 2010).

\section{Cytotoxic T Lymphocyte (CTL) Epitopes Prediction}

CTLs are $\mathrm{CD} 8^{+}$subset of T-cell responses to kill the cells having intracellular viral infections. During infection, when they encounter MHC-I mounted antigen, specific to their receptor, they transform into effector cells. Therefore, to design a vaccine, it is also crucial to predict CTL epitopes. For acquiring CTL epitopes, the selected AA sequence was uploaded on the NetCTL 1.2 (http://www.cbs.dtu.dk/services/NetCTL/) database. NetCTL 1.2 server predicts CTL epitopes in the input protein sequences and this method is established on three approaches: i) the transportation effectiveness of transporters in relation to antigen processing, ii) MHC-I attachment compatibility and iii) the proteasomal C-terminal dissociation. These three prediction outcomes are merged together, and specificity is determined from that merged score (Larsen et al., 2007). The cut-off score for identifying epitopes was prearranged at 0.75 and generally, a higher score designates higher specificity. HLA (Human Leukocyte Antigen) allele A1 supertype was selected for this prediction. The epitope prediction scores which are presented by a $<$-E sign designate that those epitopes are identified as MHC (Major Histocompatibility Complex) ligands.

\section{Multi-epitope Vaccine Construction}

To generate a multi-epitope vaccine construct, every projected BCE was chosen. Among all HTL epitopes, the ones acquiring $\mathrm{IC}_{50}$ values lesser than $50 \mathrm{nM}$ as determined by $\mathrm{NN}$ align (an artificial neural network-based method) score were selected. Overlapping HTL epitopes were merged together. Amongst the CTL epitopes, all the projected epitopes were nominated. To provide efficient separation and effective functioning, linkers have been inserted between two epitopes. $\beta$-defensin had been employed as an adjuvant. Naive T-cell and immature dendritic cells are recruited by $\beta$-defensin at the infection site and it also provides innate host response in microbial infections.

\section{Prediction of Allergenicity and Antigenicity}

To make sure the multi-epitope vaccine does not stimulate any allergenic response, the non-allergic and allergic behavior was forecasted using AlgPred (Prediction of Allergenic Proteins-http://www.imtech.res.in/raghava/algpred/) which depends on whether IgE epitopes are present on the input protein sequence. Altogether it implements four different 
standards, IgE (Immunoglobulin E) epitope + MAST (MotifAlignment and Search Tool) + SVM (Support Vector Machine) + ARPs (Allergen Representative Peptides) BLAST (Basic Local Alignment Search Tool), for forecasting of allergenicity (Saha \& Raghava, 2006a).

ANTIGENpro (http://www.scratch.proteomics.ics.uci.edu/) server was utilized for predicting the antigenicity. The predictions are established on the input sequence as well as machine learning algorithms. The accurateness of this server had been assessed to be $76 \%$ founded on cross-validation experiments (Magnan \& Baldi, 2014). To reconfirm the antigenicity of the constructed multi-epitope vaccine, we also performed antigenicity analysis using the VaxiJen server. VaxiJen (http://www.ddg-pharmfac.net/vaxijen/VaxiJen/ VaxiJen.html) is the first server for alignment-independent prediction of protective antigens. It allows antigen classification solely based on the physicochemical properties of proteins. The server performs well in cross-validations showing prediction accuracy from $70 \%$ to $89 \%$. The threshold score for both the servers was 0.4 .

\section{Physicochemical Characterization of the Vaccine Construct}

Various physicochemical features (molecular weight, sequence length, half-life, instability index, theoretical pI, aliphatic index as well as the grand average of hydropathicity or GRAVY) were further analyzed from the ProtParam tool (http://web.expasy.org/protparam/) (Gasteiger et al., 2005). Prediction results were established on the pK values of the AA.

\section{Secondary Structure Prediction}

PSIPRED (Position-Specific Iterative basic local alignment search tool based secondary structure PREDiction-http://bioinf.cs.ucl.ac.uk/index.php?id=779) server was implemented to determine the secondary structure (Jones, 1999). PSIPRED has two neural networks. The preliminary projection was achieved by its first network, which was then manipulated as input for the subsequent network. This server achieved an $81.6 \% \mathrm{Q}_{3}$ score established on the cross-validation method.

\section{Tertiary Structure Prediction and Validation}

The tertiary structure was constructed by means of the SWISS-MODEL (https://swissmodel. expasy.org) website (Waterhouse et al., 2018). The formation of the homology model contains four steps: (1) identification of templates, (2) alignment between target with the template, (3) model forming, and (4) quality assessment. The automated mode was commissioned for this to forecast the tertiary structure and was also validated from the Ramachandran plot.

\section{Molecular Docking Between Vaccine Construct and Immune Receptor}

To generate proper immune responses, the antigen along with the immune receptor has to 
work together. For this, molecular docking was performed among the immune receptors (Toll-like receptor-3/TLR-3, PDB ID: 2A0Z and Toll-like receptor-9/TLR-9, PDB ID: $3 \mathrm{WPF}$ ) with the ligand (the multi-epitope vaccine construct) with the help of PatchDock (http://bioinfo3d.cs.tau.ac.i1/PatchDock/) server. It mainly holds 3 distinctive phases; demonstration of the molecular profile, matching the surface patch, and finally filtering and then scoring (Schneidman-Duhovny et al., 2005).

\section{RESULTS}

\section{Multiple Sequence Alignment}

The multiple sequence alignment of the amino acid sequence of the HVRs of the prototype (GB strain, GenBank Accession Nos. AB330084) and the four selected hexon variants of HAdV-B3 (3Hv-1, 2, 3, and 4, GenBank Accession Nos. AF542106, DQ099432, KC456085, and AY854180 respectively) are demonstrated in Figure 1.
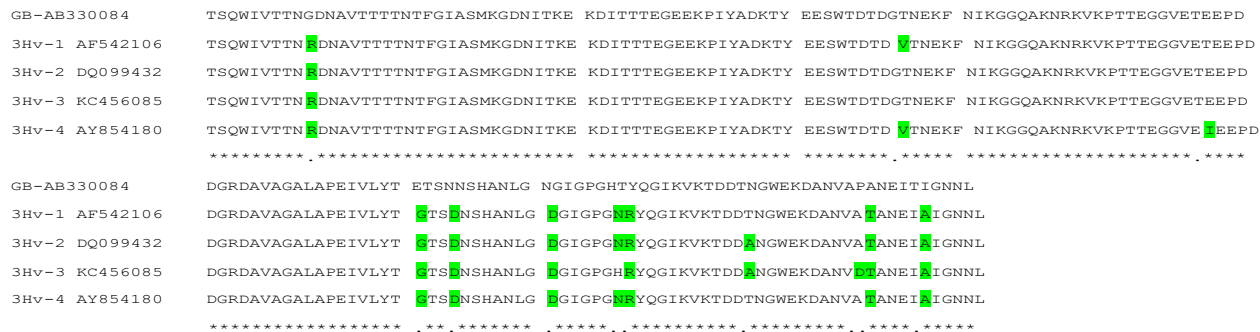

Figure 1. Multiple sequence alignment of the HVRs of the prototype (GB strain, GenBank Accession Nos. AB330084) and the four selected hexon variants of HAdV-B3 (3Hv-1, 2, 3, and 4, GenBank Accession Nos. AF542106, DQ099432, KC456085, and AY854180 respectively). The sequence variations as compared to the GB strain at different position of the HVRs are indicated with a dot at the bottom (and are also highlighted in green) and the homologies are represented by a star at the bottom.

\section{Prediction of B-Cell Epitopes (BCE), Helper T Lymphocyte (HTL), and Cytotoxic T Lymphocyte (CTL) Epitopes}

The HVRs of 3Hv-1, 2, 3, and 4 (GenBank Accession Nos. AF542106, DQ099432, $\mathrm{KC456085}$, and AY854180 respectively), were analyzed for epitope prediction.

Altogether, $8 \mathrm{BCE}$ were achieved from the HVRs of 3Hv-1 and 4, whereas, $7 \mathrm{BCE}$ were achieved from the other 2 hexon variants (3Hv-2 and 3). For those epitopes with a similar sequence amongst all four hexon variants, only one was taken into account to construct the vaccine. The overall quantity of BCE incorporated was 9.

The HTL epitopes having $\mathrm{IC}_{50}$ values lesser than $50 \mathrm{nM}$ (in accordance with $\mathrm{NN}$ align $\mathrm{IC}_{50}$ score) were selected, depicting high affinity. The final selection was done depending on 
the overlapping sequences which were then combined together to acquire the final epitopes. 5 HTL epitopes were nominated after merging the overlapping sequences.

Amongst all the achieved CTL epitopes, there were 9 from $3 \mathrm{Hv}-1$ and 8 from the other 3 hexon variations $(3 \mathrm{Hv}-2,3$, and 4$)$ that were identified as MHC ligands (presented by $\mathrm{a}<$-E sign). The different selected epitopes after merging their sequences are indicated in Table 1 and overall predictions are exhibited in supplementary data.

Table 1

The selected B-cell epitopes (BCE), HTL (Helper T Lymphocyte), and CTL (Cytotoxic T Lymphocyte) epitopes from the hypervariable regions (HVRs) of the four major hexon variant strains of $H A d V-B 3$

\begin{tabular}{lll}
\hline BCE & HTL Epitopes & CTL Epitopes \\
\hline WIVTTNR & DNFVGLMYYNSTGNMGVLAGQ & DTDVTNEKF \\
GIASMKG & DRNTELSYQLLLDSLWDRTRYFSMWKQAVDS & YTENVNLET \\
DITTTEGEE & EGEEKPIYADKTYQPEPQ & ALAPEIVLY \\
VTNEKFG & LAPEIVLYTENVNLETP & ETPDSHVVY \\
TNIKGGQAKNRKVKPTT & NTELSYQLLLDSLGDRTRYF & TSDNSHANL \\
VVYKPGTSDN & & NTELSYQLL \\
VDLQDRN & & WTDTDVTNE \\
IKVKTDDT & & GIEDELPNY \\
SWTDTDGTNEKFG & & DSLWDRTRY \\
9 BCE & 5 HTL epitopes & 9 CTL \\
& & epitopes \\
\hline
\end{tabular}

\section{Multi-epitope Vaccine Construction}

This includes 9 linear BCE, 5 HTL, and 9 CTL epitopes and is exhibited in Figure 2. Linear BCE and HTL epitopes were attached together with GPGPG and the CTL epitopes were amalgamated with AAY linkers respectively. $\beta$-defensin (GIINTLQKYYCRVRGGRCAVLSCLPKEEQIGKCSTRGRKCCRRKK), which is 45 AA long, was further added by the EAAAK linker. Following the addition of linkers and adjuvant, the final vaccine construct sequence had 415 amino acids.

\section{Allergenicity and Antigenicity Prediction}

The final multi-epitope vaccine construct was not carrying any experimentally proven IgE epitopes, hence, it was considered as non-allergic (Supplementary Data).

The antigenicity probability was 0.938684 which designates an excellent antigenic feature of the vaccine construct. Reconfirming the antigenicity data from the Vaxijen server gave us an antigenicity probability score of 0.9306 . Hence, the data from both the servers indicate that the multi-epitope vaccine construct is a good antigen. 


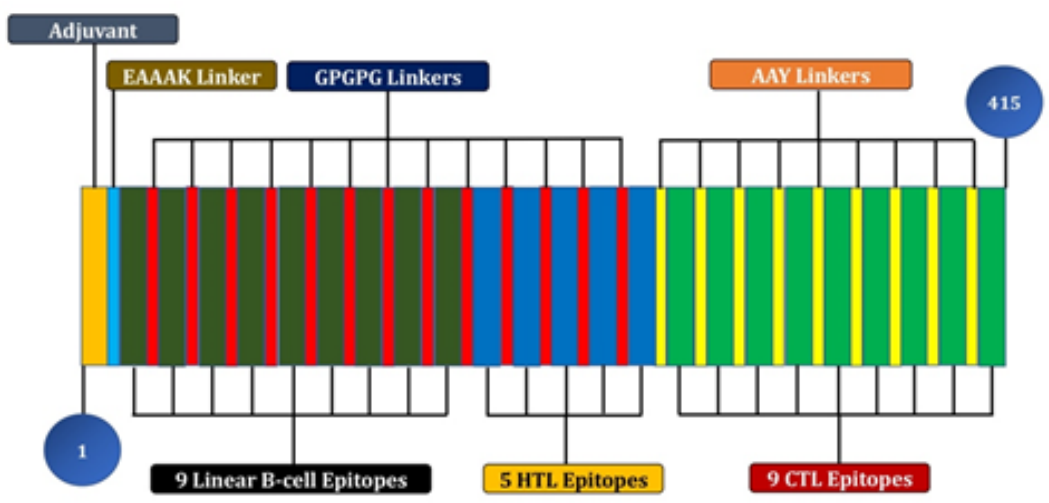

Figure 2. The multi-epitope vaccine construct consisting 9 linear (BCE) B-Cell Epitopes (dark green), 5 (HTL) Helper T Lymphocyte (blue) plus 9 (CTL) Cytotoxic T Lymphocyte (green) epitopes. The linear BCE and HTL epitopes were fused by GPGPG (red) linker and CTL epitopes were attached by AAY (yellow) linkers. $\beta$-defensin (orange) was associated with the EAAAK linker (light blue). Altogether, there were 23 different epitopes, 23 linkers, and 1 adjuvant comprising a total of 415 amino acids.

\section{Physicochemical Evaluation of the Vaccine Construct}

The molecular weight of the vaccine construct was $44 \mathrm{kDa}$ which illustrates decent antigenic characteristics. The pI (Isoelectric point) value was 4.84 which designates the vaccine construct as acidic. The anticipated half-life was 30 hours in vitro, while the anticipated half-life was more than 20 and 10 hours in yeast and E. coli respectively. The expected aliphatic index was 59.04 which designates it as thermo-stable. GRAVY was measured to be -0.687 , and the instability index was computed as 22.13 (Supplementary Data).

\section{Secondary and Tertiary Structure Estimation and Validation}

In the projected secondary structure, coil, $\alpha$-helix, and $\beta$-strands formation involved 268 AA, 86 AA and 61 AA respectively. Overall, it suggested that $64.57 \%$ were coils, $20.72 \%$ were helix, and $14.69 \%$ were stranded.

In tertiary structure prediction, 295 templates were located to match our target sequence, but the topmost 50 templates were filtered. The Global Model Quality Estimation (GMQE) score was 0.04 which reflects an accurate structure. The model achieved a QMEAN (Qualitative Model Energy ANalysis) Z-score of -2.76 which indicated the generated model was of extremely good quality. This was also highlighted by a "thumbs-up" symbol beside the score.

The Ramachandran plot showed a 79.07\% favored region and $6.98 \%$ as outliers which were highest amongst the three predicted structures. There were no bad bonds present in the structure. The MolProbity score was 2.45. The secondary structure, tertiary structure and the Ramachandran plot are displayed in Figure 3 (a, b and c respectively). 


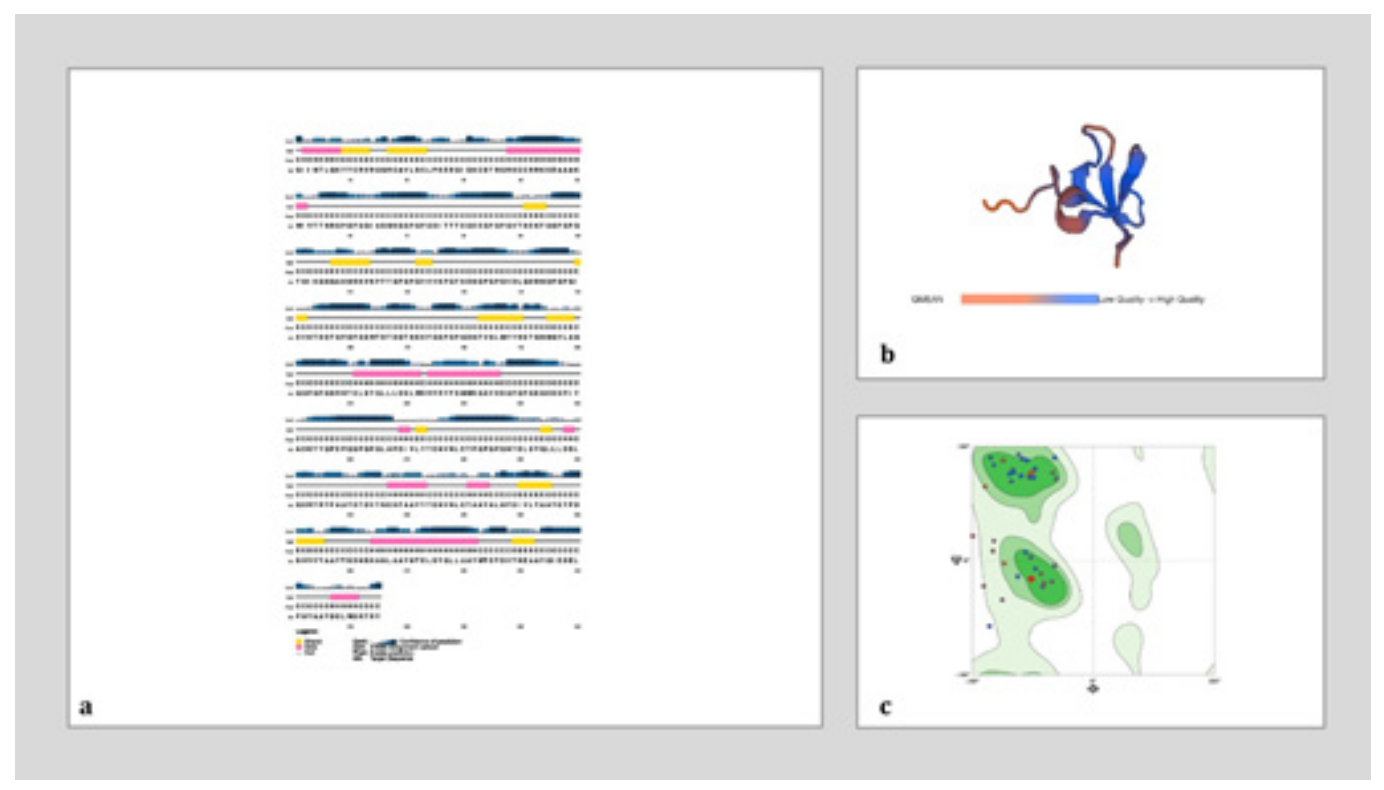

Figure 3. (a) The secondary conformational elements of the multi-epitope vaccine. The outcome indicated the organization of helix (pink colored bars), strands (yellow colored bars), and coils (black straight lines). (b) Tertiary folding of the vaccine. The orange to blue color grade bar below denotes the low quality to high quality of structure founded on QMEAN model schemes. (c) Tertiary structure validation by means of Ramachandran plot.

\section{Molecular Docking Between Immunological Receptors (Toll-Like Receptor-3 and Toll-Like Receptor-9) and the Multi-epitope Vaccine Construct}

Molecular docking generated several results among which the best 10 outcomes were sent to the FireDock server (Fast Interaction REfinement in molecular DOCKing-http://bioinfo3d. cs.tau.ac.il/FireDock/php.php) for enhancement and model number 10 was nominated as best depending on the collaborations between receptor and ligand. In Figure 4 (a) displays TLR-3 alone and (b) denotes the ligand-receptor docked complex. The geometric shape complementarity score of the best-docked model was estimated to be 12878 , the calculated interface region of interaction was 1831.80. Also, the atomic contact energy (ACE) was 457.85. The global energy (binding energy of solution) of the best-docked model was 7.44.

On the other hand, Figure 4 (c) displays TLR-9 alone and (d) denotes the ligand-receptor docked complex. The geometric shape complementarity score of the best-docked model in this case was estimated to be 13944, the calculated interface region of interaction was 2231. Also, the atomic contact energy (ACE) was 448.23. The global energy (binding energy of solution) of the best-docked model was 7.94 . 

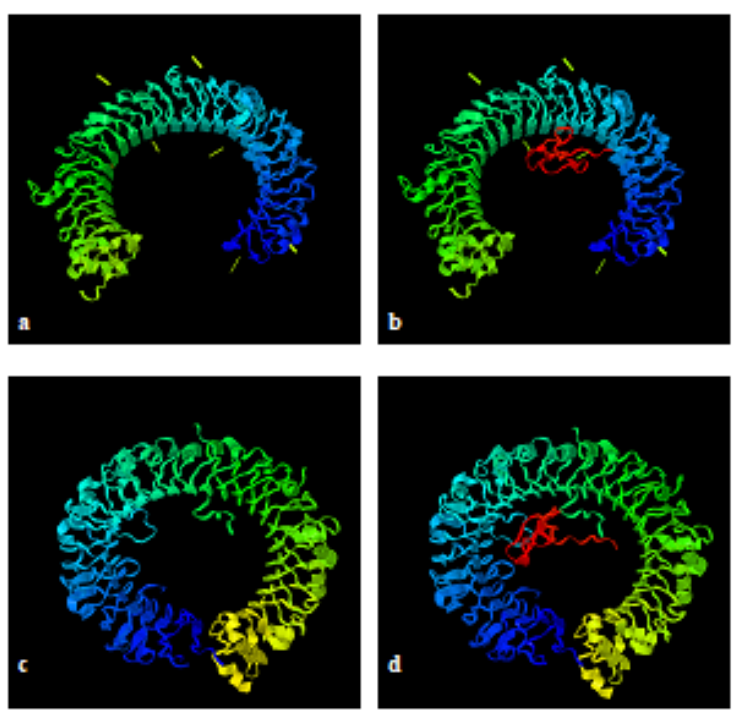

Figure 4. Comparison of Toll-like receptors-3 (TLR-3) and Toll-like receptor-9 (TLR-9) with the vaccine construct docked complex. (a) The rainbow-colored structure represents TLR-3 (PDB ID: 2A0Z), (b) The docked complex where the rainbow-colored structure is TLR-3 with the red colored structure denoting the multi-epitope vaccine construct, (c) The rainbow-colored structure represents Toll-like receptors-9/TLR-9 (PDB ID: 3WPF), and (d) The docked complex where the rainbow-colored structure is TLR-9 with the red colored structure denoting the multi-epitope vaccine construct.

\section{DISCUSSION}

HAdV-B3 causes respiratory infections with severe morbidity and mortality. It is generally considered as the most frequently identified HAdV type among pediatric age group with respiratory infections, requiring hospitalization. Over recent years, HAdV-B3 mediated respiratory illnesses have turned out as global health problem (Chen \& Tian, 2018). Henceforth, the development of a vaccine is the key to encounter this health issue.

In an effort to prevent acute respiratory disease among military personnel, a live enteric-coated oral vaccine containing strains of HAdV-E4 and B7 was introduced in 1971 (Gurwith et al., 1989). Within the period of the vaccine use, the morbidity decreased by 50-60\%, but later, many cases of HAdV-E4 and B7 mediated respiratory diseases had been detected among formerly vaccinated persons (Blasiole et al., 2004). The detailed study revealed that major antigenic changes occurred due to single or multiple coding variations in HAdV-E4 and HAdV-B7 (Crawford-Miksza et al., 1999).

HVRs of hexon of HAdV-B3 are highly diverse in their antigenic areas (Haque et al., 2018). Since the epitopes of HAdVs are conformational, changes in single or multiple AA in HVRs might considerably affect the antigenic regions (Blasiole et al., 2004). Hence, the antibody aimed at one variant may not offer a protective effect against additional existing variants of HAdV-B3. 
Considering the epidemiological and clinical significance, an effective vaccine for HAdV-B3 is in demand to curb the infection and reduce health expenses. However, there are several challenges to create a vaccine against HAdV-B3 in a traditional way, for instance, (1) unavailability of all the strains reported so far due to the lack of culturing and isolation of the virus (Purcell et al., 2007), (2) strict biosecurity rules to transport the live virus (Salerno et al., 2007), (3) the huge amount of time essential for this (Purcell et al., 2007), and finally, (4) a chance of the emergence of a new recombinant strain when multiple strains are mixed together to generate a live vaccine (Sadanand, 2011). These challenges advocate that a newer approach to vaccine development is obligatory to bypass all these difficulties. Regarding this, a multi-epitope vaccine might be suitable as it allows to include numerous epitopes. This kind of vaccine was previously developed against many viruses namely human papillomavirus (Sabah et al., 2018) and dengue (Ali et al., 2017) which showed immense diversity in their neutralizing epitopes like HAdV-B3.

In the present study, first-ever against HAdV-B3, different immunoinformatics tools were applied to develop a multi-epitope vaccine construct. For this, different epitopes were predicted and a vaccine construct of 415 AA length was built carrying 9 linear BCE, 5 HTL, and 9 CTL epitopes conjugated with linkers. $\beta$-defensin was combined as an adjuvant since the peptides themselves are inadequately immunogenic. It recruits naive T-cell and immature dendritic cells at the site of infection through the CCR6 receptor (C-C Chemokine Receptor type 6), and provides adaptive and innate host response (Mohan et al., 2013).

The molecular weight of the newly developed vaccine construct was $44 \mathrm{kDa}$ which implied excellent antigenic properties as some higher molecular weight vaccines showed severe side effects. Previously, whole cells were used as vaccine candidates which were effective but some serious side effects were also associated. A well-known example is the vaccine against Bordetella pertussis having multiple pertussis antigens, where the molecular weight of the filamentous hemagglutinin and pertactin were $200 \mathrm{kDa}$ and $69 \mathrm{kDa}$ respectively (Kanra et al., 1993). The pI (Isoelectric point) value of the construct was 4.84 which denotes an acidic quality (Gasteiger et al., 2005). The $\mathrm{pI}$ of a protein is a good indicator of the protein's solubility as proteins tend to be the least soluble near their pI (Righetti et al., 1981). The Grand average of hydropathicity (GRAVY) value of our construct was -0.687 which designates hydrophilicity of the protein. The structure of the vaccine construct was stable since the instability index was 22.13 . The aliphatic index of a protein is defined by the relative volume occupied by the aliphatic side chains (alanine, valine, isoleucine, and leucine) and high aliphatic index may be regarded as a positive factor for the increase of the thermostability (Ikai, 1980). The aliphatic index of the multi-epitope vaccine construct was 59.04 signifying that it is thermostable.

TLR-3 recognizes dsRNA, and thus, is involved in antiviral host immune responses (Alexopoulou et al., 2001). As dsRNA can potentially be generated during adenoviral 
infection (Zhu et al., 2007), we have performed the docking of the multi-epitope vaccine construct with TLR-3. Moreover, TLR-3 has been shown to promote cross-priming to virusinfected cells (Schulz et al., 2005). The best-docked model with TLR-3 had a geometric shape complementarity score of 12878 and the global energy score was 7.44. However, HAdV-B3 activates innate immunity by its dsDNA through TLR-dependent pathway and the recognition of HAdV-B3 by the plasmacytoid dendritic cells is mediated by TLR-9 (Iacobelli-Martinez \& Nemerow, 2007). Therefore, we had also performed the docking analysis of the multi-epitope vaccine construct with TLR-9. The best-docked model with TLR-9 had a geometric shape complementarity score of 13944 and the global energy score was 7.94 .

Lastly, we believe that our newly proposed multi-epitope vaccine construct against HAdV-B3 could offer several advantages such as, (1) focusing an immune reaction concerning only the key neutralizing epitopes while circumventing the induction of antibodies that has no protective role and may even be harmful, (2) enable a more robust immune response capable of potentially accommodating mutations in neutralizing epitopes, (3) evading the hazard of handling live viruses, (4) it could be produced readily in the laboratory, (5) it can diminish the chances of co-infection as every possible epitope would be present and finally (6) if new hexon variants of HAdV-B3 appear in future, their epitopes could be easily incorporated in the existing vaccine.

\section{CONCLUSION}

Considering the heterogeneity of HAdV-B3 and the complexity of generating conventional vaccines, a multi-epitope vaccine construct incorporating all epitopes of four major HAdV-B3 hexon variants was built utilizing various immunoinformatics tools. We believe that this in silico multi-epitope vaccine construct will considerably reduce the time and expense of biological work essential for vaccine development with a better possibility of getting the desired solution by lessening the necessity for countless trial and error repeats of assays.

\section{ACKNOWLEDGEMENT}

A part of this project is supported by the Fundamental Research Grant Scheme (FRGS/1/2017/SKK11/AIMST/02/1) offered by the Ministry of Higher Education, Malaysia.

\section{REFERENCES}

Alexopoulou, L., Holt, A. C., Medzhitov, R., \& Flavell, R. A. (2001). Recognition of double-stranded RNA and activation of NF-кB by Toll-like receptor 3. Nature, 413(6857), 732-738. doi: https://doi. org/10.1038/35099560 
Ali, M., Pandey, R. K., Khatoon, N., Narula, A., Mishra, A., \& Prajapati, V. K. (2017). Exploring dengue genome to construct a multi-epitope based subunit vaccine by utilizing immunoinformatics approach to battle against dengue infection. Scientific Reports, 7(1), 1-13. doi: 10.1038/s41598-017-09199-w.

Blasiole, D. A., Metzgar, D., Daum, L. T., Ryan, M. A. K., Wu, J., Wills, C., ... \& Russell, K. L. (2004). Molecular analysis of adenovirus isolates from vaccinated and unvaccinated young adults. Journal of Clinical Microbiology, 42(4), 1686-1693. doi: 10.1128/JCM.42.4.1686-1693.2004

Chen, S., \& Tian, X. (2018). Vaccine development for human mastadenovirus. Journal of Thoracic Disease, 10(Suppl 19), S2280-S2294. doi: 10.21037/jtd.2018.03.168.

Crawford-Miksza, L. K., Nang, R. N., \& Schnurr, D. P. (1999). Strain variation in adenovirus serotypes 4 and 7a causing acute respiratory disease. Journal of Clinical Microbiology, 37(4), 1107-1112. doi: 10.1128/ JCM.37.4.1107-1112.1999

Dong, Y. S. (2005). Unite and devote to the works of pediatric clinical virology. Zhonghua Er Ke Za Zhi= Chinese Journal of Pediatrics, 43(1), 1-2.

Gasteiger, E., Hoogland, C., Gattiker, A., Duvaud, S., Wilkins, M. R., Appel, R. D., \& Bairoch, A. (2005). Protein identification and analysis tools on the ExPASy Server. In J. M. Walker (Ed.), The Proteomics Protocols Handbook (pp. 571-607). Totowa, New Jersey: Humana Press. doi: 10.1385/1-59259-890-0:571.

Gurwith, M. J., Horwith, G. S., Impellizzeri, C. A., Davis, A. R., Lubeck, M. D., \& Hung, P. P. (1989). Current use and future directions of adenovirus vaccine. Seminars in Respiratory Infections, 4(4), 299-303.

Haque, E., Banik, U., Monowar, T., Anthony, L., \& Adhikary, A. K. (2018). Worldwide increased prevalence of human adenovirus type $3(\mathrm{HAdV}-3)$ respiratory infections is well correlated with heterogeneous hypervariable regions (HVRs) of hexon. PloS One, 13(3), 1-12. doi: 10.1371/journal.pone.0194516.

Harley, D., Harrower, B., Lyon, M., \& Dick, A. (2001). A primary school outbreak of pharyngoconjunctival fever caused by adenovirus type 3. Communicable Diseases Intelligence, 25(1), 9-12.

Iacobelli-Martinez, M., \& Nemerow, G. R. (2007). Preferential activation of Toll-like receptor nine by CD46utilizing adenoviruses. Journal of Virology, 81(3), 1305-1312. doi: 10.1128/JVI.01926-06

Ikai, A. (1980). Thermostability and aliphatic index of globular proteins. The Journal of Biochemistry, 88(6), 1895-1898. doi: https://doi.org/10.1093/oxfordjournals.jbchem.a133168

Jones, D. T. (1999). Protein secondary structure prediction based on position-specific scoring matrices. Journal of Molecular Biology, 292(2), 195-202. doi: 10.1006/jmbi.1999.3091.

Kanra, G., Ceyhan, M., Vandevoorde, D., \& Bogaerts, H. (1993). Acellular pertussis diphtheria-tetanus-pertussis vaccine containing separately purified pertussis toxoid, filamentous haemagglutinin and $69 \mathrm{kDa}$ outer membrane protein as a booster in children. European Journal of Pediatrics, 152(6), 478-483. doi: https:// doi.org/10.1007/BF01955054

Lai, C. Y., Lee, C. J., Lu, C. Y., Lee, P. I., Shao, P. L., Wu, E. T., ... \& Lin, J. J. (2013). Adenovirus serotype 3 and 7 infection with acute respiratory failure in children in Taiwan, 2010-2011. PloS One, 8(1), 1-7. doi: 10.1371/journal.pone.0053614. 
Larsen, M. V., Lundegaard, C., Lamberth, K., Buus, S., Lund, O., \& Nielsen, M. (2007). Large-scale validation of methods for cytotoxic T-lymphocyte epitope prediction. BMC Bioinformatics, 8(1), 1-12. doi: 10.1186/1471-2105-8-424.

Lion, T. (2014). Adenovirus infections in immunocompetent and immunocompromised patients. Clinical Microbiology Reviews, 27(3), 441-462. doi:10.1128/CMR.00116-13

Liu, T., Fan, Y., Li, X., Gu, S., Zhou, Z., Xu, D., ... \& Tian, X. (2018). Identification of adenovirus neutralizing antigens using capsid chimeric viruses. Virus Research, 256, 100-106. doi: 10.1016/j.virusres.2018.08.007.

Lynch, J. P., \& Kajon, A. E. (2016). Adenovirus: Epidemiology, global spread of novel serotypes, and advances in treatment and prevention. Seminars in Respiratory and Critical Care Medicine, 37(4), 586-602. doi: 10.1055/s-0036-1584923.

Magnan, C. N., \& Baldi, P. (2014). SSpro/ACCpro 5: Almost perfect prediction of protein secondary structure and relative solvent accessibility using profiles, machine learning and structural similarity. Bioinformatics, 30(18), 2592-2597. doi: 10.1093/bioinformatics/btu352.

Mohan, T., Sharma, C., Bhat, A. A., \& Rao, D. N. (2013). Modulation of HIV peptide antigen specific cellular immune response by synthetic $\alpha$ - and $\beta$-defensin peptides. Vaccine, 31(13), 1707-1716. doi: https://doi. org/10.1016/j.vaccine.2013.01.041.

Panda, S., Banik, U., \& Adhikary, A. K. (2020). Bioinformatics analysis reveals four major hexon variants of human adenovirus type-3 (HAdV-3) as the potential strains for development of vaccine and siRNAbased therapeutics against HAdV-3 respiratory infections. Infection, Genetics and Evolution, 85, 1-9. doi: 10.1016/j.meegid.2020.104439.

Pichla-Gollon, S. L., Drinker, M., Zhou, X., Xue, F., Rux, J. J., Gao, G. P., ... \& Bergelson, J. M. (2007). Structure-based identification of a major neutralizing site in an adenovirus hexon. Journal of Virology, 81(4), 1680-1689. doi: 10.1128/JVI.02023-06.

Purcell, A. W., McCluskey, J., \& Rossjohn, J. (2007). More than one reason to rethink the use of peptides in vaccine design. Nature reviews Drug discovery, 6(5), 404-414. doi: https://doi.org/10.1038/nrd2224

Righetti, P. G., Tudor, G., \& Ek, K. (1981). Isoelectric points and molecular weights of proteins: A new table. Journal of Chromatography A, 220(2), 115-194. doi: https://doi.org/10.1016/S0021-9673(00)88456-3

Sabah, S. N., Gazi, M. A., Sthity, R. A., Husain, A. B., Quyyum, S. A., Rahman, M., \& Islam, M. R. (2018). Designing of epitope-focused vaccine by targeting E6 and E7 conserved protein sequences: An immunoinformatics approach in Human Papilloma Virus 58 isolates. Interdisciplinary Sciences, Computational Life Sciences, 10(2), 251-260. doi: 10.1007/s12539-016-0184-5.

Sadanand, S. (2011). Vaccination: the present and the future. The Yale Journal of Biology and Medicine, 84(4), 353-359.

Saha, S., \& Raghava, G. P. S. (2006a). AlgPred: Prediction of allergenic proteins and mapping of IgE epitopes. Nucleic Acids Research, 34(Web Server issue), W202-209. doi: 10.1093/nar/gk1343. 
Saha, S., \& Raghava, G. P. S. (2006b). Prediction of continuous B-cell epitopes in an antigen using recurrent neural network. Proteins, 65(1), 40-48. doi: 10.1002/prot.21078.

Salerno, R. M., Gaudioso, J., \& Brodsky, B. H. (2007). Laboratory biosecurity handbook. Boca Raton, Florida: CRC press.

Schulz, O., Diebold, S. S., Chen, M., Näslund, T. I., Nolte, M. A., Alexopoulou, L., ... \& e Sousa, C. R. (2005). Toll-like receptor 3 promotes cross-priming to virus-infected cells. Nature, 433(7028), 887-892. doi: https://doi.org/10.1038/nature03326

Schneidman-Duhovny, D., Inbar, Y., Nussinov, R., \& Wolfson, H. J. (2005). PatchDock and SymmDock: Servers for rigid and symmetric docking. Nucleic Acids Research, 33(Web Server issue), W363-W367. doi: 10.1093/nar/gki481.

Wang, P., Sidney, J., Kim, Y., Sette, A., Lund, O., Nielsen, M., \& Peters, B. (2010). Peptide binding predictions for HLA DR, DP and DQ molecules. BMC Bioinformatics, 11(1), 1-12. doi: 10.1186/1471-2105-11-568.

Waterhouse, A., Bertoni, M., Bienert, S., Studer, G., Tauriello, G., Gumienny, R., ... \& Schwede, T. (2018). SWISS-MODEL: Homology modelling of protein structures and complexes. Nucleic Acids Research, 46(W1), W296-W303. doi: 10.1093/nar/gky427.

Zhu, J., Huang, X., \& Yang, Y. (2007). Innate immune response to adenoviral vectors is mediated by both Toll-like receptor-dependent and-independent pathways. Journal of Virology, 81(7), 3170-3180. doi: 10.1128/JVI.02192-06 
Somnath Panda, Urmila Banik and Arun Kumar Adhikary

\section{SUPPLEMENTARY DATA}

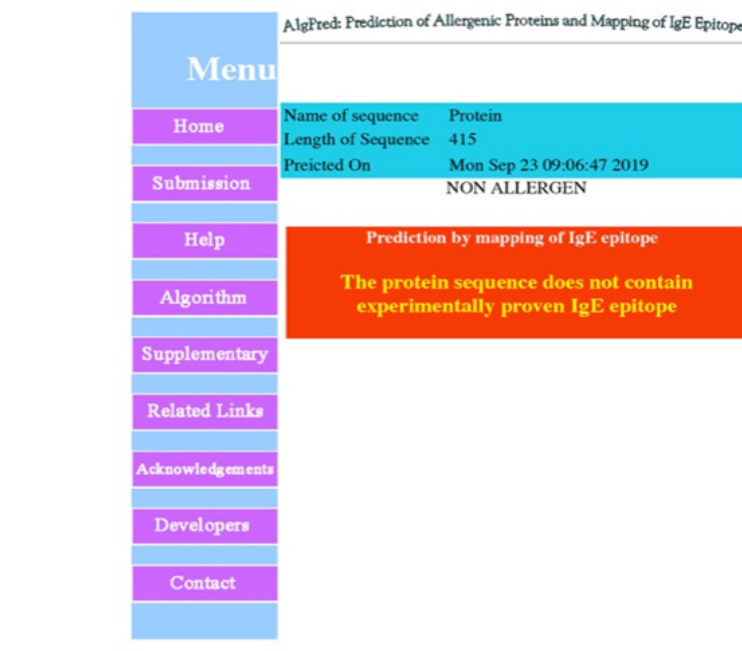

Exasy moperam wed

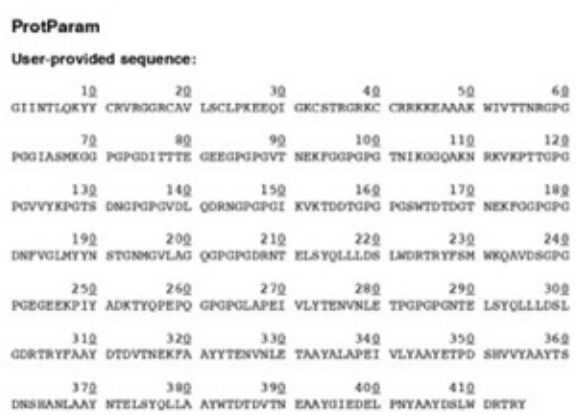


In Silico Vaccine Against Human Adenovirus Type B3

Eossy noparam neet

$1809 / 2019.5233 \mathrm{me}$

Total number of negativoly eharged residues (Asp + a1u) , 51 Total number of positively eharged residves (arg + Lys) it 3 il

Atomie conposition:

carbon

Nitrogen \& 531

$\begin{array}{lll}\text { Oxygen } & 0 & 632 \\ \text { Sultur } & 8 & 10\end{array}$

Pormula: $\mathrm{C}_{1951} \mathrm{H}_{2979} \mathrm{~N}_{531} \mathrm{O}_{632} \mathrm{~S}_{16}$

rotal number of atoms: 6103

Extinction coeffieients!

Extinction coefficients are in units of $\mathrm{N}^{-1} \mathrm{~cm}^{-1}$, at $280 \mathrm{nin}$ messured in vater.

Ext. coeftieient 73605 , assuning all pairs of cys residves form cystines
Abs $0.11(=1 \mathrm{~g} / 1) \quad 1.661$,

Ext. coetifieient 73230

NDS $0.11(=1 \mathrm{~g} / 1) \quad 1.653$, assuming all cys residoes are reduced

Estimated balf-11fe:

The N-terminal of the sequence considered is $G$ (G1y).

The estimated malf-life ist 30 bours (mamalian reticulocytes, in vitro),

$\rightarrow 10$ hours (Escherichia ooli, in vivo).

\section{Instability index:}

The instability index (II) is conpoted to be 22.13

This elassifies the protein as stable.

Aliphatie indexi 59.04

Grand average of hydropathieity (CRAvr) : -0.687 
The predictiow of linear B-cell epitopes as obtained from the hnpenariable regions $(H V R s)$ of the four major heron variant strains of $H A d V-B 3$ by using the BCEPRED senver

\begin{tabular}{|c|c|c|}
\hline Strain & Predicted linear B-cell epitopes & $\begin{array}{c}\text { Positions in HVR (132- } \\
450 \mathrm{AA})\end{array}$ \\
\hline \multirow[t]{8}{*}{$3 \mathrm{Hv} \cdot 1$} & WIVTTNR & $135-141$ \\
\hline & GLASMKG & $153-159$ \\
\hline & DITTTEGEE & $172-180$ \\
\hline & VTNEKFG & $205-211$ \\
\hline & TNIKGGQAKNRKVKPTT & $232-248$ \\
\hline & VVYKPGTSDN & $294-303$ \\
\hline & VDLQDRN & $352-358$ \\
\hline & IKVKTDDT & $422-429$ \\
\hline \multirow[t]{7}{*}{$3 \mathrm{Hv} \cdot 2$} & WIVTTNR & $135-141$ \\
\hline & GLASMKG & $153-159$ \\
\hline & DITTTEGEE & $172-180$ \\
\hline & SWTDTDGTNEKFG & $199-211$ \\
\hline & TNIKGGQAKNRKVKPTT & $232-248$ \\
\hline & VVYKPGTSDN & $294-303$ \\
\hline & VDLQDRN & $352-358$ \\
\hline \multirow[t]{7}{*}{$3 \mathrm{Hv} \cdot 3$} & WTVTTNR & $135-141$ \\
\hline & GLASMKG & $153-159$ \\
\hline & DITTTEGEE & $172-180$ \\
\hline & SWTDTDGTNEKFG & $199-211$ \\
\hline & TNIKGGQAKNRKVKPTT & $232-248$ \\
\hline & VVYKPGTSDN & 294-303 \\
\hline & VDLQDRN & $352-358$ \\
\hline \multirow[t]{8}{*}{$3 \mathrm{Hv}-4$} & WTVTTNR & $135-141$ \\
\hline & GLASMKG & $153-159$ \\
\hline & DITTTEGEE & $172-180$ \\
\hline & VTNEKFG & $205-211$ \\
\hline & TNIKGGQAKNRKVKPTT & $232-248$ \\
\hline & VVYKPGTSDN & $294-303$ \\
\hline & VDLQDRN & $352-358$ \\
\hline & IKVKTDDT & $422-429$ \\
\hline
\end{tabular}

The prediction of Helper $T$ Lywphocite $(H T L$ ) pitopes as abtained from the hopenvariable ngions (HVRs) of the four major hexon variant strains of $H A d V$. $B 3$ by asing she IEDB senver

\begin{tabular}{|c|c|c|c|c|}
\hline \multicolumn{5}{|c|}{$3 \mathrm{Hv}-1$} \\
\hline HTL Epitopes & $\begin{array}{c}\text { Positions } \\
\text { in IVVR } \\
(132- \\
450)\end{array}$ & Allele & $\begin{array}{c}\text { Percentile } \\
\text { Rank }\end{array}$ & $\mathrm{IC}_{\mathrm{se}}(\mathrm{nMI})$ \\
\hline VGLMYYNSTGNMGVL & 327.341 & $\begin{array}{c}\text { HLA- } \\
\text { DRB1*07:01 }\end{array}$ & 1.10 & 12.60 \\
\hline GLMYYNSTGNMGVLA & 328.342 & $\begin{array}{c}\text { HLA- } \\
\text { DRB } 1^{*} 07: 01\end{array}$ & 1.30 & 14.00 \\
\hline FVGLMYYNSTGNMGV & $327-340$ & $\begin{array}{c}\text { HLA- } \\
\text { DRB } 1 * 07: 01\end{array}$ & 1.80 & 17.10 \\
\hline LMYYNSTGNMGVLAG & $329 \cdot 343$ & $\begin{array}{c}\text { HLA- } \\
\text { DRB } 1^{*} 07 ; 01\end{array}$ & 2.20 & 19.50 \\
\hline RNTELSYQLLLDSLW & 357.371 & $\begin{array}{c}\text { HLA- } \\
\text { DRB4*01:01 }\end{array}$ & 0.87 & 25.30 \\
\hline FVGLMYYNSTGNMGV & $326-340$ & $\begin{array}{c}\text { HLA- } \\
\text { DRB } 1^{*} 15: 01\end{array}$ & 1.40 & 25.60 \\
\hline NFVGLMYYNSTGNMG & $325-339$ & $\begin{array}{c}\text { HLA- } \\
\text { DRB1*15:01 }\end{array}$ & 1.50 & 25.70 \\
\hline DNFVGI.MYYNSTGNM & $324-338$ & $\begin{array}{c}\text { HLA- } \\
\text { DRB1*15:01 }\end{array}$ & 1.50 & 26.30 \\
\hline VGLMYYNSTGNMGVL. & 327.341 & $\begin{array}{c}\text { HLA- } \\
\text { DRB } 1^{*} 15: 01\end{array}$ & 1.70 & 28.50 \\
\hline NTELSYQLL.LDSLWD & $358-372$ & $\begin{array}{c}\text { HLA- } \\
\text { DRB } 4^{*} 01: 01\end{array}$ & 1.20 & 29.00 \\
\hline MYYNSTGNMGVLAGQ & $330-344$ & $\begin{array}{l}\text { HLA- } \\
\text { DRB1*07:01 }\end{array}$ & 3.50 & 29.20 \\
\hline DRNTELSYQLLLDSL & $356-370$ & $\begin{array}{c}\text { HLA- } \\
\text { DRB } 4^{*} 01: 01\end{array}$ & 1.20 & 29.90 \\
\hline GEEKPIYADKTYQPE & $178-192$ & $\begin{array}{c}\text { HLA- } \\
\text { DRB3*01:01 }\end{array}$ & 1.40 & 31.00 \\
\hline TELSYQLLLDSLWDR & 359.373 & $\begin{array}{c}\text { HLA- } \\
\text { DRB4*01:01 }\end{array}$ & 1.60 & 34.60 \\
\hline EEKPIYADKTYQPEP & $179-193$ & $\begin{array}{c}\text { HLA- } \\
\text { DRB3*01:01 }\end{array}$ & 1.60 & 36.00 \\
\hline LWDRTRYFSMWKQAV & $370-384$ & $\begin{array}{c}\text { HLA- } \\
\text { DRBS*01:01 }\end{array}$ & 5.50 & 36.40 \\
\hline
\end{tabular}


In Silico Vaccine Against Human Adenovirus Type B3

\begin{tabular}{|c|c|c|c|c|}
\hline WDRTRYFSMWKQAVD & $371-385$ & $\begin{array}{c}\text { HL.A- } \\
\text { DRB5 }\end{array}$ & 5.60 & 37.00 \\
\hline EKPIYADKTYQPEPQ & $180-194$ & $\begin{array}{l}\text { HLA- } \\
\text { DRB3*01:01 }\end{array}$ & 1.80 & 38.70 \\
\hline APEIVLYTENVNLET & $275-289$ & $\begin{array}{c}\text { HILA- } \\
\text { DRB1*15:01 }\end{array}$ & 2.60 & 38.90 \\
\hline DRTRYFSMWKQAVDS & $372-386$ & $\begin{array}{l}\text { HLA- } \\
\text { DRB5*01:01 }\end{array}$ & 6.10 & 40.70 \\
\hline PEIVLYTENVNLETP & $276-290$ & $\begin{array}{c}\text { HLA- } \\
\text { DRB1*15:01 }\end{array}$ & 3.00 & 43.90 \\
\hline LAPEIVLYTENVNLE & $274-288$ & $\begin{array}{l}\text { HLA- } \\
\text { DRB1*15:01 }\end{array}$ & 3.40 & 48.30 \\
\hline EGEEKPIYADKTYQP & $177 \cdot 191$ & $\begin{array}{c}\text { HLA- } \\
\text { DRB3*01:01 }\end{array}$ & 2.10 & 49.60 \\
\hline GLMYYNSTGNMGVLA & $328-342$ & $\begin{array}{l}\text { HILA- } \\
\text { DRB } 1 * 15 ; 01\end{array}$ & 3.50 & 49.70 \\
\hline \multicolumn{5}{|c|}{$3 H \mathrm{Hv}-2$} \\
\hline HTL Epitopes & $\begin{array}{c}\text { Positiens } \\
\text { in HVR } \\
(132- \\
450)\end{array}$ & Allele & $\begin{array}{l}\text { Percentile } \\
\text { Rank }\end{array}$ & ICse(nN) \\
\hline VGLMYYNSTGNMGVL & 327.341 & $\begin{array}{c}\text { HLA- } \\
\text { DRB1 }{ }^{*} 07.01\end{array}$ & 1.10 & 12.60 \\
\hline GLMYYNSTGNMGVLA & 328.342 & $\begin{array}{c}\text { HLA- } \\
\text { DRBI } 07.01\end{array}$ & 1.30 & 14.00 \\
\hline FVGLMYYNSTGNMGV & $327-340$ & $\begin{array}{c}\text { HL.A- } \\
\text { DRB1*07.01 }\end{array}$ & 1.80 & 17.10 \\
\hline LMYYNSTGNMGVLAG & $329-343$ & $\begin{array}{c}\text { HLA- } \\
\text { DRB1*07:01 }\end{array}$ & 2.20 & 19.50 \\
\hline RNTELSYQLLLDSLG & 357.371 & $\begin{array}{c}\text { HLA- } \\
\text { DRB4*01:01 }\end{array}$ & 0.84 & 24.80 \\
\hline FVGLMYYNSTGNMGV & $326 \cdot 340$ & $\begin{array}{c}\text { HLA- } \\
\text { DRBI*15.01 }\end{array}$ & 1.40 & 25.60 \\
\hline NFVGLMYYNSTGNMG & $325-339$ & $\begin{array}{c}\text { HL.A- } \\
\text { DRBI*15:01 }\end{array}$ & 1.50 & 25.70 \\
\hline DNFVGLMYYNSTGNM & $324-338$ & $\begin{array}{c}\text { HL.A- } \\
\text { DRB1*15.01 }\end{array}$ & 1.50 & 26.30 \\
\hline VGLMYYNSTGNMGVL. & 327.341 & $\begin{array}{c}\text { HLA- } \\
\text { DRBI*15:01 }\end{array}$ & 1.70 & 28.50 \\
\hline MYYNSTGNMGVLAGQ & $330-344$ & $\begin{array}{c}\text { HL.A- } \\
\text { DRB1 }{ }^{* 07.01}\end{array}$ & 3.50 & 29.20 \\
\hline NTELSYQLLLDSLGD & $358-372$ & $\begin{array}{c}\text { HLA- } \\
\text { DRB4*01:01 }\end{array}$ & 1.20 & 29.40 \\
\hline DRNTELSYQL.L.DSL. & $356-370$ & $\begin{array}{c}\text { HL.A- } \\
\text { DRB4*01:01 }\end{array}$ & 1.20 & 29.90 \\
\hline GEEKPTYADKTYQPE & $178-192$ & $\begin{array}{c}\text { HLA- } \\
\text { DRB3*01:01 }\end{array}$ & 1.40 & 31.00 \\
\hline TELSYQLLLDSLGDR & $359-373$ & $\begin{array}{c}\text { HL.A- } \\
\text { DRB4*01:01 }\end{array}$ & 1.70 & 35.50 \\
\hline EEKPIYADKTYQPEP & 179.193 & $\begin{array}{c}\text { HLA- } \\
\text { DRB3*01:01 }\end{array}$ & 1.60 & 36.00 \\
\hline EKPIYADKTYQPEPQ & $180-194$ & $\begin{array}{c}\text { HILA- } \\
\text { DRB3*01:01 }\end{array}$ & 1.80 & 38.70 \\
\hline APEIVLYTENVNLET & $275-289$ & $\begin{array}{c}\text { HLA- } \\
\text { DRB1*15.01 }\end{array}$ & 2.60 & 38.90 \\
\hline SYQLLLLDSLGDRTRY & $362-376$ & $\begin{array}{c}\text { HL.A- } \\
\text { DRB1 *03.01 }\end{array}$ & 1.60 & 40.10 \\
\hline YQLLLDSLGDRTRYF & $363-377$ & $\begin{array}{c}\text { HLA- } \\
\text { DRBI }{ }^{* 03.01}\end{array}$ & 1.70 & 42.50 \\
\hline PETVLYTENVNLETP & $276-290$ & $\begin{array}{c}\text { HI.A- } \\
\text { DRB1*15:01 }\end{array}$ & 3.00 & 43.90 \\
\hline LAPEIVLYTENVNLE & $274-288$ & $\begin{array}{c}\text { HLA- } \\
\text { DRB1*15:01 }\end{array}$ & 3.40 & 48.30 \\
\hline EGEEKPIYADKTYQP & $177-191$ & $\begin{array}{c}\text { HI_A- } \\
\text { DRB3*01:01 }\end{array}$ & 2.10 & 49.60 \\
\hline GLMYYNSTGNMGVLA & 328.342 & $\begin{array}{c}\text { HI.A- } \\
\text { DRB!*15:01 }\end{array}$ & 3.50 & 49.70 \\
\hline
\end{tabular}




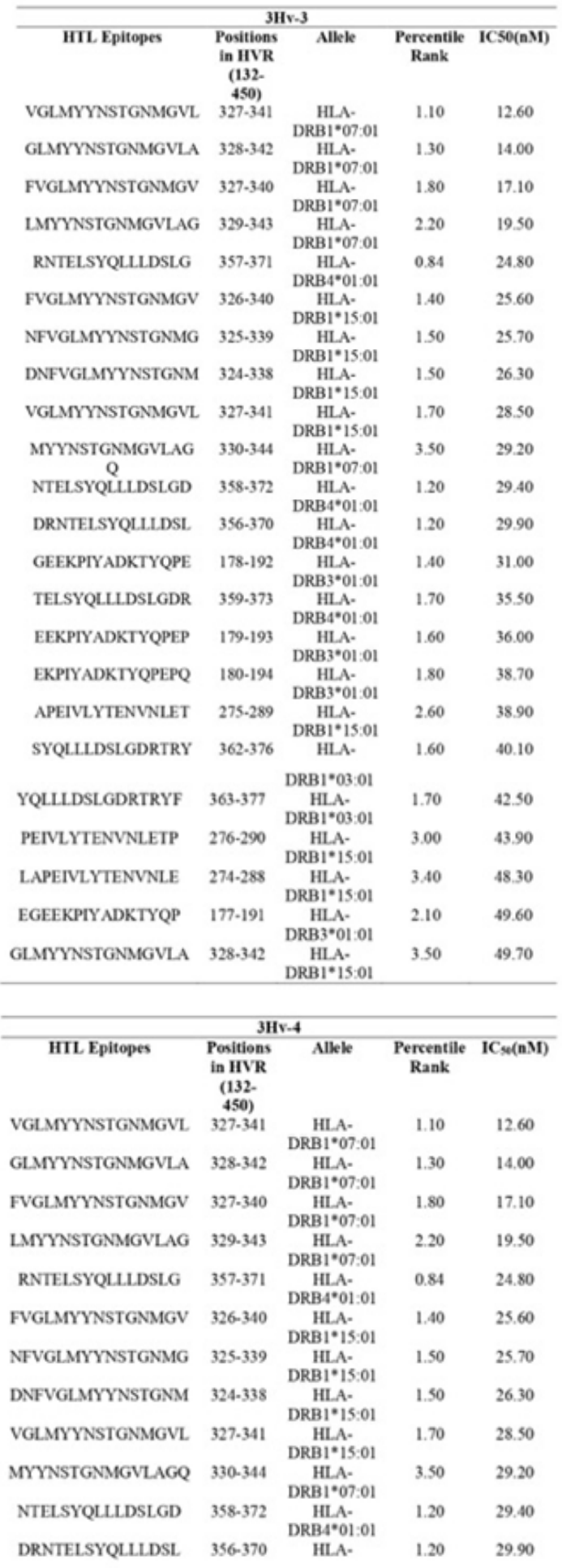




\begin{tabular}{|c|c|c|c|c|}
\hline GEEKPTYADKTYQPE & $178-192$ & $\begin{array}{c}\text { DRB4*01:01 } \\
\text { HL_A- } \\
\text { DRB3*01:01 }\end{array}$ & 1.40 & 31.00 \\
\hline TELSYQLLLDSLGDR & $359-373$ & $\begin{array}{c}\text { HLA- } \\
\text { DRBA } 4^{*} 01: 01\end{array}$ & 1.70 & 35.50 \\
\hline EEKPTYADKTYQPEP & 179.193 & $\begin{array}{c}\text { HLA- } \\
\text { DRB3*01:01 }\end{array}$ & 1.60 & 36.00 \\
\hline EKPIYADKTYQPEPQ & $180-194$ & $\begin{array}{c}\text { HLA- } \\
\text { DRB3*01:01 }\end{array}$ & 1.80 & 38.70 \\
\hline APEIVLYTENVNLET & $275-289$ & $\begin{array}{c}\text { HLA- } \\
\text { DRB1*15:01 }\end{array}$ & 2.60 & 38.90 \\
\hline SYQLLLDSLGDRTRY & $362-376$ & $\begin{array}{c}\text { HL_A- } \\
\text { DRB1*03:01 }\end{array}$ & 1.60 & 40.10 \\
\hline YQLLLDSLGDRTRYF & $363-377$ & $\begin{array}{c}\text { HLA- } \\
\text { DRB1*03:01 }\end{array}$ & 1.70 & 42.50 \\
\hline PETVLYTENVNLETP & $276-290$ & $\begin{array}{l}\text { HL_A- } \\
\text { DRB1*15:01 }\end{array}$ & 3.00 & 43.90 \\
\hline LAPEIVLYTENVNLE & $274-288$ & $\begin{array}{c}\text { HLA- } \\
\text { DRB1*15:01 }\end{array}$ & 3.40 & 48.30 \\
\hline EGEEKPIYADKTYQP & $177 \cdot 191$ & $\begin{array}{c}\text { HLA- } \\
\text { DRB3*01:01 }\end{array}$ & 2.10 & 49.60 \\
\hline GLMYYNSTGNMGVLA & $328-342$ & $\begin{array}{c}\text { HL_A- } \\
\text { DRB1*15:01 }\end{array}$ & 3.50 & 49.70 \\
\hline
\end{tabular}

The prediction of CTL epitopes based on MHC-I binding as obtained from the hypenvariable regions (HVRs) of the four major hexou variant strains of HAdV. $B 3$ by using the NetCTL 1.2 server

\begin{tabular}{|c|c|c|c|}
\hline $\begin{array}{c}\text { Hexon } \\
\text { Variant }\end{array}$ & CTL Epitopes & $\begin{array}{c}\text { Positions in IVVR } \\
(132-450 \mathrm{AA})\end{array}$ & $\begin{array}{c}\text { Combined } \\
\text { (COMB) Score }\end{array}$ \\
\hline \multirow[t]{9}{*}{$3 H \mathrm{v}-1$} & DTDVTNEKF & $202-210$ & 20685 \\
\hline & YTENVNLET & $281-289$ & 1.9503 \\
\hline & ALAPEIVLY & 273.281 & 1.6845 \\
\hline & ETPDSHVVY & $288-296$ & 1.6837 \\
\hline & TSDNSHANL. & $300-308$ & 1.3163 \\
\hline & NTELSYQLL. & $358-366$ & 1.1167 \\
\hline & WTDTDVTNE & $200-208$ & 0.9420 \\
\hline & GIEDELPNY & $398-406$ & 0.8811 \\
\hline & DSLWDRTRY & $368-376$ & 0.7810 \\
\hline \multirow[t]{8}{*}{$3 \mathrm{Hv}-2$} & YTENVNLET & $281-289$ & 1.9503 \\
\hline & DTDGTNEKF & $202 \cdot 210$ & 1.6860 \\
\hline & ALAPEIVLY & $273-281$ & 1.6845 \\
\hline & ETPDSHVVY & $288-296$ & 1.6837 \\
\hline & TSDNSHANL & $300-308$ & 1.3163 \\
\hline & NTELSYQL.L. & $358-366$ & 1.1350 \\
\hline & WTDTDGTNE & $200-208$ & 0.9130 \\
\hline & GIEDELPNY & $398-406$ & 0.8811 \\
\hline \multirow[t]{8}{*}{$3 \mathrm{Hv}-3$} & YTENVNIET & $281-289$ & 1.9503 \\
\hline & DTDGTNEKF & $202-210$ & 1.6860 \\
\hline & ALAPEIVLY & $273-281$ & 1.6845 \\
\hline & ETPDSHVVY & $288-296$ & 1.6837 \\
\hline & TSDNSHANL & $300-308$ & 1.3163 \\
\hline & NTELSYQLL. & $358-366$ & 1.1350 \\
\hline & WTDTDGTNE & $200-208$ & 0.9130 \\
\hline & GIEDELPNY & $398-406$ & 0.8811 \\
\hline \multirow[t]{8}{*}{$3 \mathrm{Hv}-4$} & DTDVTNEKF & $202-210$ & 2.0685 \\
\hline & YTENVNLET & 281.289 & 1.9503 \\
\hline & ALAPEIVLY & $273-281$ & 1.6845 \\
\hline & ETPDSHVVY & $288-296$ & 1.6837 \\
\hline & TSDNSHANL & $300-308$ & 1.3163 \\
\hline & NTELSYQLL & $358-366$ & 1.1350 \\
\hline & WTDTDVTNE & $200-208$ & 0.9420 \\
\hline & GIEDELPNY & $398-406$ & 0.8811 \\
\hline
\end{tabular}


